\title{
EFECTOS DE LA CONTAMINACIÓN HÍDRICA SOBRE LA SALUD PÚBLICA DE LA POBLACIÓN DE LA CUENCA COATA, DE LA REGIÓN DE PUNO - 2019
}

\author{
Julio Cesar QUISPE MAMANI ${ }^{1}$ \\ Hugo Rubén MARCA MAQUERA ${ }^{2}$ \\ Vladimir Ylich Felipe MAMANI SONCO 3 \\ Ronald Raúl ARCE COAQUIRA ${ }^{4}$
}

\begin{tabular}{|lcc|}
\hline Recibido & $:$ & 20.03 .2020 \\
Aceptado & $:$ & 02.06 .2020 \\
Publicado & $:$ & 06.07 .2020 \\
\hline
\end{tabular}

RESUMEN: El objetivo de la presente investigación es analizar los efectos de la contaminación hídrica sobre la salud pública de la población Cuenca-Coata. La metodología aplicada fue el método cuantitativo; la población de estudio comprende toda la Cuenca Coata, afectados por la contaminación hídrica; para lo cual se utilizó encuestas, entrevistas y otras fuentes. Las enfermedades identificadas son es su mayoría digestivas, respiratorias e infecciosas, lo cual conlleva a que la población involucrada tenga reducidos niveles de productividad, afectando a los ingresos familiares y una inadecuada calidad de vida, toda vez que las enfermedades incrementan en la zona de estudio; el 75\% de la población en análisis se ve afectada por la contaminación hídrica de la Cuenca Coata, de los cuales el $21 \%$ asisten a los principales centros de salud para tratar los malestares generados por la misma. Además, el gasto promedio por salud de un peruano es de 20-100 soles mensuales; pero a causa del consumo de aguas contaminadas, este gasto tiende a incrementarse en un 20 a $30 \%$, por lo que, la cuenca pasa por una transición de factores de riesgos ambientales causados por la acumulación de residuos sólidos y por medio de las aguas residuales vertidos en los principales ríos de la Cuenca.

Palabras claves: Contaminación hídrica, Cuenca Coata, residuos sólidos, salud pública

\section{Effects of water pollution on the public health of the population of the Coata watershed, of the Puno region - 2019}

\begin{abstract}
The objective of this research is to analyze the effects of water pollution on the public health of the Cuenca-Coata population. The applied methodology was the quantitative method; the study population includes the entire Coatawatershed, affected by water pollution; For which, surveys, interviews and other sources were used. The diseases identified are mostly digestive, respiratory and infectious, which means that the population involved has reduced levels of productivity, affecting family income and an inadequate quality of life, since the diseases increase in the study area. ; 75\% of the population under analysis is affected by water pollution in the Coatawatershed, of which $21 \%$ attend the main health centers to treat the discomforts generated by it. In addition, the average health expenditure of a Peruvian is 20-100 soles per month; but due to the consumption of contaminated water, this expenditure tends to increase by 20 to $30 \%$, therefore, the watershed goes through a transition of environmental risk factors caused by the accumulation of solid waste and by means of wastewater discharges into the main rivers of the watershed.
\end{abstract}

Keywords: Water pollution, Coatawatershed, solid waste, public health.

\footnotetext{
${ }^{1}$ Universidad Nacional del Altiplano. Email: jcesarqm@yahoo.es

${ }^{2}$ Universidad Nacional de Moquegua. Email: lugo 1121@hotmail.com

${ }^{3}$ Universidad Nacional del Altiplano. Email: vyf-apolo@ hotmail.com

${ }^{4}$ Universidad Andina Néstor Cáceres Velásquez. Email: arceroni1@ @otmail.com
} 


\section{Journal of the Academy $|2|$}

\section{INTRODUCCIÓN}

La contaminación es uno de los problemas más grandes que existen en el planeta y el más peligroso, ya que, al destruir la tierra y su naturaleza original, termina por destruir a las mismas personas que lo habitan. Uno de los principales problemas es la contaminación hídrica que por lo general es causada directa o indirectamente por las acciones del ser humano (Samboni, Carvajal \& Escobar, 2007). Esta provoca que el agua se vuelva peligrosa tanto para su consumo como para uso en general, ya sea a nivel personal o industrial. Así mismo, el agua contaminada también resulta peligrosa para el resto de las especies de animales y para las plantas y prácticamente todas las formas de vida que dependan de ella (Mayta, 1999).

Según el Instituto Geológico y Minero de España (2003); en su informe menciona que una de las consecuencias del crecimiento demográfico y la concentración urbana es la contaminación ambiental; en donde se ha podido confirmar que cuando la densidad poblacional era pequeña, se garantizaba la integridad del agua; sin embargo, actualmente el incremento de la población, el cual demanda mayor cantidad de alimento, energía y servicios, generando así el desequilibrio del recurso hídrico (Zegarra, 2014). Es decir que la contaminación del agua puede ser originada de forma natural o artificial, debido al desarrollo y a la industrialización del hombre, por lo que la generación de residuos es cada vez mayor y gran parte de ellos terminan en los ríos, haciendo que estas se contaminen, por lo tanto, la contaminación hídrica en su mayoría es de origen antropogénico.

El crecimiento poblacional genera mayor deterioro ambiental del agua en las fuentes naturales, así como el vertimiento de aguas residuales domésticas sin o con deficiente tratamiento; y además por el desarrollo de la actividad industrial, agraria y ganadera del hombre; rompiendo así el equilibrio de los ecosistemas y la armonía entre éstos y el hombre (Comisión económica del agua, 2011).

El impacto de la población, en temas de contaminación, sobre los sistemas ecológicos del planeta se ha ido incrementando en los últimos años, poniendo de manifiesto la relación existente entre los niveles de contaminación ambiental y la salud pública, en donde las enfermedades infecciosas representan un gran riesgo y son la principal causa de muerte en la población de estudio (Bofill et al., 2005). Por otro lado, uno de los factores que afecta al recurso hídrico es la falta de condiciones sanitarias básicas, lo que obliga a los habitantes a hacer sus necesidades básicas a cielo abierto, además, a esta problemática se le suma el uso indiscriminado de agroquímicos que causan la contaminación de las aguas superficiales provocando el deterioro cada vez mayor de las microcuencas (CENRE, 1999).

La contaminación de la cuenca Coata es preocupante tanto para la salud pública como para el desarrollo del país, ya que este problema retrasa las iniciativas de mejora de calidad de vida, así mismo este se ha convertido en un problema social, por ende, será necesario realizar un estudio detallado, de los cuales se identificaran los principales efectos que tiene la contaminación hídrica de la cuenca Coata sobre la salud pública para así brindar alternativas de solución eficientes y adecuadas al medio geográfico (Encarnación,1985).Se debe resaltar que la contaminación hídrica de la cuenca Coata genera diferentes tipos de enfermedades, así como las de tipos gastrointestinales, infecciones en la piel, cólera, hepatitis A, fiebre, entre otros; las cuales en su mayoría generan la disminución de la esperanza de vida del poblador (Rodríguez, García\& García, 2016).El recurso hídrico; ya sean de ríos, lagos o mar pueden contaminarse por agentes biológicos y causar un grupo de enfermedades que son clasificadas como infectocontagiosas o transmisibles. De manera general son causadas por microorganismos pertenecientes a los grupos conocidos como virus, bacterias, hongos, parásitos, etc (Amaya, 2005).

La salud pública es la ciencia y el arte de prevenir las dolencias y discapacidades, prolongar la vida, fomentar la salud y la eficiencia física y mental, mediante esfuerzos organizados por las autoridades locales y nacionales para mejorar la calidad del medio ambiente, como también controlar enfermedades infecciosas y no infecciosas, así como las lesiones, educar al individuo en los principios de la higiene personal, organizar los servicios para el diagnóstico y el tratamiento de las enfermedades y para la rehabilitación, como también desarrollar políticas que aseguren a cada miembro de la comunidad un 


\section{Journal of the Academy $|3|$}

nivel de vida adecuado para el mantenimiento de la salud, es decir, llegar al bienestar social y desarrollo sostenible de la población puesto que una persona saludable es mucho más productiva y eficiente (Olmeda, 2006).Por otro lado, a causa de la contaminación hídrica que afecta la salud pública, se generan altos gastos para el tratamiento de las diferentes enfermedades adquiridas de la población involucrada, además del gasto generado por parte del gobierno, debido a que la salud pública es un factor importante para el desarrollo sostenible de un país. El gasto promedio mensual por salud de un peruano es de 59 soles; pero a causa del consumo de aguas contaminadas, este gasto tiende a incrementarse en un 20\% (Loayza \& Cano, 2015).

En este sentido el objetivo de la presente investigación es analizar los efectos de la contaminación hídrica sobre la salud pública de la población en la Cuenca Coata, la cual busca mejorar la calidad del recurso hídrico, contribuyendo así a la disminución de los problemas relacionados con las salud de las familias en la zona de estudio, por lo que consecuentemente conllevara a una disminución del gasto destinado a la salud, de tal manera se llegara a conseguir a una adecuada distribución de los ingresos destinados a las necesidades básicas y de esta contribuir a una mejora en la calidad de vida de los pobladores que residen en la cuenca Coata

\section{DESARROLLO}

\begin{tabular}{|c|c|c|c|}
\hline $\begin{array}{l}\text { Existe un alto grado de } \\
\text { contaminación hídrica } \\
\text { que afecta a la salud de } \\
\text { la población } \\
\text { involucrada. }\end{array}$ & $\begin{array}{l}\text { P.G. ¿Cuáles son los } \\
\text { efectos de la } \\
\text { contaminación hídrica } \\
\text { sobre la salud pública } \\
\text { de la población de la } \\
\text { cuenca Coata? } \\
\text { P.1. ¿Cuáles son los } \\
\text { principales factores de } \\
\text { la contaminación } \\
\text { hídrica que afectan a la } \\
\text { salud de las familias de } \\
\text { la Cuenca Coata? } \\
\text { P.2. ¿Cuáles son los } \\
\text { problemas de salud que } \\
\text { existen a causa de la } \\
\text { contaminación de la } \\
\text { cuenca Coata? ida qué manera } \\
\text { P.3. ¿De la } \\
\text { repercute } \\
\text { contaminación hídrica } \\
\text { en el gasto en salud? }\end{array}$ & $\begin{array}{l}\text { O.G. Analizar los } \\
\text { efectos de la } \\
\text { contaminación hídrica } \\
\text { sobre la salud pública } \\
\text { de la población } \\
\text { afectada en la cuenca } \\
\text { Coata en el año } 2019 . \\
\text { O.E.1. Determinar los } \\
\text { principales factores de } \\
\text { la contaminación } \\
\text { hídrica de la Cuenca } \\
\text { Coata. Identificar los } \\
\text { O.E.2. In salud a } \\
\text { problemas de la } \\
\text { causa de hán } \\
\text { contaminación hídrica } \\
\text { de la Cuenca Coata. } \\
\text { O.E.3. Determinar la } \\
\text { repercusión de la } \\
\text { contaminación hídrica } \\
\text { sobre los gastos en } \\
\text { salud (población y el } \\
\text { gobierno). }\end{array}$ & $\begin{array}{l}\text { H.G. La contaminación } \\
\text { hídrica de la Cuenca } \\
\text { Coata influye de } \\
\text { manera negativa a la } \\
\text { salud pública de las } \\
\text { poblaciones aledañas. } \\
\text { H.1. Los } \\
\text { contaminantes más } \\
\text { importantes que } \\
\text { afectan de manera } \\
\text { negativa y significativa } \\
\text { a la salud de la } \\
\text { población son; } \\
\text { residuos sólidos, } \\
\text { vertimiento de aguas } \\
\text { servidas, y metales } \\
\text { pesados. } \\
\text { H.2. Las principales } \\
\text { enfermedades y/o } \\
\text { malestares a causa de } \\
\text { la contaminación } \\
\text { hídrica son las } \\
\text { digestivas } \\
\text { infecciosas. } \\
\text { H.3. La contaminación } \\
\text { hídrica repercute de } \\
\text { manera directa sobre } \\
\text { los gastos en salud. }\end{array}$ \\
\hline
\end{tabular}

\section{Materiales y método \\ Metodología de la investigación}

El diseño metodológico aplicado en la presente investigación es de tipo no experimental de corte transversal (Hernández, Fernández \& Baptista, 2010). La investigación se desarrollará con un enfoque descriptivo, debido a que los datos serán obtenidos directamente de la realidad problemática, y considerando las distintas fuentes de información sin que estas sean modificadas o alteradas, serán 


\section{Journal of the Academy $|4|$}

obtenidas tal como se presentan al momento de la aplicación de los instrumentos de recolección de datos a las personas integrantes de la Cuenca del Rio Coata. (Kerlinger, 1979:116).

\section{Técnicas e instrumentos para obtener información}

Para el desarrollo de la presente investigación se aplicará instrumentos de recolección de datos que medirán los índices e indicadores de las variables de estudio, se utilizará la técnica de la encuesta, planificada, corregida y preparada de acuerdo a los objetivos planteados, para finalmente, en la fase de sistematización de la información, toda la información generada en el campo será sometido a un procesamiento estadístico de la información y de análisis respectivo con la finalidad de implementar el diagnóstico de la realidad, se empleará la estadística descriptiva (Cazau,2006). También se empleó la distribución de frecuencias, para el caso de las enfermedades se revisó los datos registrados por los Centros de Salud que se encuentran ubicadas por las zonas de la Cuenca (Hernández, Fernández \& Baptista, 2010).

\section{Población y tamaño de la muestra}

La población está constituida por la población total de los siguientes distritos del departamento de Puno:

Tabla 2. Población de la Cuenca Coata

\begin{tabular}{llll}
\hline Distritos & Rural & Urbano & Total \\
\hline Santa Lucia & 1,759 & 5,697 & 7,456 \\
Paratia & 2,903 & 0 & 2,903 \\
Palca & 1,930 & 0 & 1,930 \\
Lampa & 5,946 & 5,695 & 11,641 \\
Vilavila & 1,308 & 0 & 1,308 \\
Cabanillas & 2,240 & 2,404 & 4,644 \\
Cabanilla & 5,848 & 0 & 5,848 \\
Juliaca & 12,088 & 223,022 & 235,110 \\
Cabana & 5,215 & 0 & 5,215 \\
Caracoto & 7,119 & 0 & 7,119 \\
Pusi & 5,311 & 0 & 5,311 \\
Huata & 3,302 & 0 & 3,302 \\
Coata & 6,941 & 0 & 6,941 \\
\hline Total & 61,910 & 236,818 & $\mathbf{2 9 8 , 7 2 8}$ \\
\hline
\end{tabular}

Fuente: INEI Censos 2017, Elaboración propia

Para el cálculo del tamaño de la muestra se utilizó la fórmula que se presenta en el estudio de Loyola y Soncco (2006).

$$
\frac{N K^{2} E_{a} E_{0}}{(N-1) e^{2}+\left(K^{2} E_{a} E_{a}\right)}
$$

Donde:

$N=$ Tamaño poblacional.

$e=$ Es el error máximo admitido.

$K=$ Es el coeficiente de confianza.

$E a=$ Probabilidad de que la población puede enfermarse por causa de la mala calidad de agua para su consumo.

$E o=$ Probabilidad de que la población puede enfermarse por otras causas.

Para hallar la muestra se asumió que (Ea) de ocurrencia de un SI y a la probabilidad (Eo) de ocurrencia de un NO sean iguales (50 \%) lo cual garantiza un mayor tamaño posible de la muestra (Cazau, 2006).

Para el caso en estudio el cálculo del tamaño de la muestra es: 


\section{Journal of the Academy $|5|$}

$$
\begin{gathered}
N=298,728, e=5 \%(95 \% \text { de N.C }), K=1.96, E_{a}=50 \%, E_{0}=50 \% \\
n=\frac{298,728 * 1,96^{2} * 0.5 * 0.5}{(298,728-1) 0,05^{2}+\left(1,96^{2} * 0.5 * 0.5\right)} \\
n=383.72
\end{gathered}
$$

La muestra de la investigación es de 384 habitantes de la cuenca Coata.

\section{Modelos econométricos \\ Modelo CLOGLOG}

Modelo CLOGLOG para la determinación de los problemas de salud a causa de la contaminación hídrica de la Cuenca Coata y el nivel económico de la población afectada de la Cuenca Coata (Ravelo \& Balaguer, 2019).

Se estimará un modelo cloglog para determinar la probabilidad de que las personas expuestas a la contaminación de la Cuenca Coata presenten un síntoma o enfermedad. En este sentido, la ecuación a estimar está dada por:

$$
P_{i}=P(\text { presentan algun sitoma o enfermedad }=1)=f(t)
$$

La ecuación (1) describe la probabilidad de presentar algún síntoma o enfermedad, que se representara por el 1. Como alternativa, los modelos de respuesta binaria cualitativa recuperan la eficiencia en los estimadores (Ravelo\& Balaguer,2019); de este por:

$$
\begin{gathered}
p_{i}=F\left(x_{i}^{\prime} \beta\right) \\
F(t)=1-(e)^{-e^{t}} \\
\text { donde } \mathrm{t}=x_{i}^{\prime} \beta
\end{gathered}
$$
modo, el modelo estimable estará dado

Por lo tanto, el modelo se presenta de la siguiente manera:

$$
p_{i}=F\left(x_{i}^{\prime} \beta\right)=F(t)=1-(e)^{-e^{t}}
$$

Se espera que quienes permanezcan en promedio mayor tiempo en la zona o hagan mayor uso del recurso hídrico contaminado tengan mayor probabilidad de sufrir un síntoma o una enfermedad (Viera, 2019). Es de interés determinar el efecto marginal que el cambio en las variables independientes aporta a la probabilidad de presentar un síntoma leve o enfermedad. La medición de este efecto implica derivar la ecuación (1), respecto de los regresores $(X)$. La ecuación (3) expresa el efecto marginal.

$$
F(t)=\frac{\exp (t)}{1+\exp (t)}
$$

Para corroborar la robustez del modelo, se empleó el método de adición de variables (al adicionar variables los coeficientes no evidenciaran cambios significativos) y se realizaran pruebas de ajuste de los diferentes modelos, validando su consistencia y robustez. La presencia de síntomas o enfermedades en los individuos encuestados se debe a la exposición a la contaminación o a condiciones preexistentes. Se evaluará las condiciones socioeconómicas de la población involucrada teniendo en consideración el nivel de ingresos, si accede a algún centro de salud (público o privado) y cuál es el porcentaje de gasto que invierte en su recuperación (Viera, 2019).

Los gastos totales incluyen: gasto en movilidad, gasto en medicamentos (o remedios caseros) y gastos en tratamiento. 


\section{Journal of the Academy $|6|$}

\section{Modelo LOGIT}

El modelo logit permite obtener estimaciones de la probabilidad de un suceso e identificar los factores de riesgo que determinan dichas probabilidades. Este modelo es empleado cuando se desea comparar un grupo de variables independientes con una variable dependiente no métrica (dicotómica), por lo que su posible respuesta es 0 (Fracaso, Ausencia de) o 1 (Éxito, Presencia de), por este motivo no se puede usar un modelo lineal (Ravelo\& Balaguer,2019).

Sea la variable respuesta $Y$, la cual sólo puede tomar los valores $Y=1$ (presencia de la característica de interés) con probabilidad de ocurrencia igual a $\pi$ y $Y=0$ (ausencia de la característica de interés) con probabilidad 1- $\pi$. Además, sea la covariable (variable predictiva o variable independiente) $\mathrm{X}$, la cual puede ser categórica o continua (Viera, 2019).

Si la variable $\mathrm{Y}$ es el resultado de un experimento de Bernoulli, esto es, las observaciones son independientes, entonces la variable aleatoria tiene distribución de Bernoulli, c con:

$$
E(Y / X=x)=\pi \text { (Esperanza condicional de } \mathrm{Y} \text { dado } \mathrm{X}=\mathrm{x}), \mathrm{y}
$$

\section{$V(Y / X=x)=\pi(1-\pi)$ (Varianza condicional de $\mathrm{Y}$ dado $\mathrm{X}=\mathrm{x})$.}

Por lo tanto, la probabilidad de que $\mathrm{Y}=1$ es igual a la $\boldsymbol{E}(\boldsymbol{Y} / \boldsymbol{X}=\boldsymbol{x})=\pi$ y puede ser calculada a partir de una distribución de probabilidad que tiene la forma de la curva sigmoidea, en particular esta curva puede ser la logística (Viera, 2019).

$$
E(Y / X=x)=\pi=\frac{e^{\beta_{0}+\beta_{1} X}}{1+e^{\beta_{0}+\beta_{1} X}} .
$$

Donde $\boldsymbol{\eta}=\boldsymbol{\beta} \mathbf{0}+\boldsymbol{\beta} \mathbf{1} \boldsymbol{X}$ es el predictor lineal y la función de enlace canónico es:

$$
\theta=\ln \left(\frac{E(Y)}{1-E(Y)}\right)=\ln \left(\frac{\pi}{1-\pi}\right) \text {. }
$$

Su representación como un modelo lineal generalizado, será:

$$
\operatorname{logit}(\pi)=\ln \left(\frac{E(Y / X=x)}{1-E(Y / X=x)}\right)=\ln \left(\frac{\pi(x)}{1-\pi(x)}\right)=\beta_{0}+\beta_{1} X .
$$

\section{Modelo Probit}

A diferencia de la regresión logit que utiliza una función de enlace del tipo, logaritmo natural de los Odds Ratio, la función de enlace Probit es la inversa de una distribución normal estándar acumulada ( $\mathrm{N}$ $(0,1)$ (Ravelo\& Balaguer,2019).

La función de distribución acumulada de la normal estándar $(\Phi)$ se expresa:

$$
g=\Phi(\eta)=P(Z \leq \eta)=\frac{1}{\sqrt{2 \pi}} \int_{-\infty}^{\eta} e^{-\frac{t^{2}}{2}} d t
$$

Donde:

Sea la variable $\eta=X^{T} \beta$ respuesta $\mathrm{Y}$, la cual sólo puede tomar los valores $\mathrm{Y}=1$ (presencia de la característica de interés) con probabilidad de ocurrencia igual a $\pi$. Luego, el modelo de regresión 


\section{Journal of the Academy $|7|$}

Probit se expresa como (Viera, 2019):

Para linealizar la relación aplicamos la transformación inversa:

$$
\pi=E(Y / x)=\Phi(\eta)=\Phi\left(x^{T} \beta\right) \quad \Phi^{-1}(\pi)=\Phi^{-1}\left(E\left(\frac{Y}{x}\right)\right)=\eta=x^{T} \beta
$$

Donde $\boldsymbol{\eta}=\boldsymbol{x} \boldsymbol{T} \boldsymbol{\beta}$ es el predictor lineal y la función de enlace canónico es:

$$
\theta=\phi^{-1}(\pi)=\Phi^{-1}(E(Y))
$$

Su representación como un modelo lineal generalizado, será

$$
\operatorname{probit}(\pi)=\Phi^{-1}(\pi)=\Phi^{-1}(E(Y / x))=X^{T} \beta
$$

El modelo Probit permite llevar a cabo estudios en donde se cumple la existencia de una variable latente en donde se observa evidencia dicotómica (Viera, 2019).

\section{Resultados y discusión}

\section{Principales factores de la contaminación hídrica de la Cuenca Coata}

Cabe resaltar que dentro de los resultados de la encuesta realizada en la Cuenca Coata, los encuestados respondieron que el factor químico era una de los principales contaminantes de la Cuenca Coata, mientras que los otros dos (factores físicos y biológicos) no tenían repercusión significativa en la contaminación hídrica de la Cuenca, por ende, se consideró a los factores químicos (residuos sólidos, aguas residuales, detergentes, fertilizantes, aceites y metales pesados) como las variables

\begin{tabular}{|c|c|c|c|c|c|c|c|}
\hline Probit regre & & & & Number & $=0 \mathrm{bs}$ & $=$ & 384 \\
\hline & & & & Wald cl & $2(4)$ & $=$ & 432.44 \\
\hline & & & & Prob > & i2 & $=$ & 0.0000 \\
\hline Log pseudolit & ihood $=-24$ & 322492 & & Pseudo & & $=$ & 0.8492 \\
\hline & & Robust & & & & & \\
\hline $\mathrm{CH}$ & Coef. & Std. Err. & z & $\mathrm{P}>|\mathrm{z}|$ & {$[95 \%$} & Conf. & Interval] \\
\hline RS & 7.243448 & .7308328 & 9.91 & 0.000 & 5.811 & 1042 & 8.675854 \\
\hline AR & 2.116809 & .4495826 & 4.71 & 0.000 & 1.235 & 5644 & 2.997975 \\
\hline DETER & 1.034295 & .4424962 & 2.34 & 0.019 & .1670 & 0179 & 1.901571 \\
\hline MT & 4.907388 & .2948138 & 16.65 & 0.000 & 4.329 & 9563 & 5.485212 \\
\hline _cons & -7.000168 & .8979703 & -7.80 & 0.000 & -8.760 & 0157 & -5.240178 \\
\hline
\end{tabular}
independientes del modelo (Tabla 3).

Tabla 3. Resultados de la regresión probit - Objetivo específico 1

Note: 37 failures and 71 successes completely determined.

- Para la estimación del modelo fueron necesarias 10 iteraciones

- El valor de "Prob>chi2 = 0.0000" representa el valor de P para el test de la razón de verosimilitud que prueba la hipótesis nula $(\mathrm{H} 0)$ de que todos los coeficientes beta del modelo (los efectos) son igual a cero, tomando en consideración un nivel de confianza del $95 \%$ se acepta la hipótesis nula del modelo 1

- La interpretación del "Pseudo R2" de McFadden (similar a la interpretación del R2 tradicional) indica que aproximadamente el $84.92 \%$ de la variación de contaminación hídrica (variable dependiente) puede ser explicada por la variación de las variables independientes del modelo (AR, RS, DETER y MT). El modelo se ajusta bien cuando el pseudo R2 se aproxima a uno, en este caso específicamente el modelo se ajusta bien.

- Luego en la tabla se observa " $\mathrm{P}<$ lzl", se observa el valor $\mathrm{P}$ del test que prueba la hipótesis nula, de que el coeficiente de cada variable es igual a cero. En este caso, con un $95 \%$ de 


\section{Journal of the Academy $|8|$}

confianza aceptamos la $\mathrm{H} 0$ para todas las variables del modelo, debido a que todas presentan datos menores a 0.05 .

- Para las cuatro variables independientes, la relación con la contaminación hídrica $(\mathrm{CH})$ es directa, debido a que los signos de los coeficientes de cada una de las variables independientes (RS, AR, DETR y MT) son positivas. Cabe resaltar que los coeficientes solo muestran la relación que tiene la variable dependiente con la variable independiente.

- Los valores "z" prueban si las variables independientes analizadas son significativas, si dicho valor es mayor a 1.96 (con un nivel de confianza del 95 por ciento) prueba que cada coeficiente es diferente de cero y para rechazar dicho valor este debe de ser menor 1.96. Los valores más altos de " $\mathrm{z}$ " significan que existe mayor relevancia de las variables, cabe resaltar que los valores del " $z$ " tienen que ser mayores a 1.96 en valor absoluto para que el modelo mantenga dichas variables (significativas). En esta regresión específicamente se puede observar que todos los valores de "z" son mayores a 1.96, lo que significa que todas las variables independientes son significativas.

La probabilidad en promedio de que si exista contaminación hídrica en a Cuenca Coata es de aproximadamente 99.32 por ciento (Tabla 4).

Tabla 4. Efecto marginal - Objetivo específico 1

\begin{tabular}{|c|c|c|c|c|c|c|c|}
\hline \multicolumn{8}{|c|}{$\begin{aligned} \text { Marginal effects after probit } \\
y=\operatorname{Pr}(\mathrm{CH}) \text { (predict) } \\
=.9931679\end{aligned}$} \\
\hline variable & $d y / d x$ & Std. Err & z & $P>|z|$ & $95 \%$ & C.I. & $\mathrm{x}$ \\
\hline$R S *$ & .9995721 & .00049 & 2052.87 & 0.000 & .998618 & 1.00053 & .875 \\
\hline$A R *$ & .191456 & .06175 & 3.10 & 0.002 & .070422 & .31249 & .755208 \\
\hline DETER* & .0273503 & .01975 & 1.39 & 0.166 & -.01135 & .066051 & .552083 \\
\hline $\mathrm{MT} *$ & .0658415 & .03805 & 1.73 & 0.084 & -.008737 & .14042 & .195313 \\
\hline
\end{tabular}

La Salida de resultados nos permite ver en la columna "P>z" en donde se observa el valor $\mathrm{P}$ del test que prueba la hipótesis nula, de que el coeficiente beta de cada variable es igual a cero. En este caso, con un $95 \%$ de confianza aceptamos la $\mathrm{H}_{0}$ para las dos primeras variables independientes (Residuos Sólidos y Aguas Residuales), debido a que todas presentan datos menores a 0.05. Mientras que el uso de detergentes (DETER) y metales pesados (MT), luego de aplicado el efecto marginal, resultaron ser no significativas para el modelo.

Los coeficientes resultantes de la estimación del modelo reflejan los efectos marginales promedio $(\mathrm{dy} / \mathrm{dx})$ de cada variable independiente sobre la probabilidad de respuesta, los cuales nos indican lo siguiente:

- Al existir residuos sólidos que tengan un contacto directo o indirecto con la Cuenca Coata (RS), la probabilidad de que exista contaminación hídrica $(\mathrm{CH})$ incrementa en promedio 99.96 puntos porcentuales.

- La probabilidad de que exista contaminación hídrica incrementa en 19.15 puntos porcentuales cuando las aguas residuales (AR) tienen contacto con el rio de la Cuenca Coata.

- La probabilidad de que exista contaminación hídrica incrementa en 2.74 puntos porcentuales cuando los detergentes (DETER) son vertidos en la Cuenca Coata.

- Cuando los metales pesados (MT) entran en contacto con las aguas de la Cuenca Coata, la probabilidad de que exista contaminación hídrica $(\mathrm{CH})$ incrementa en 6.58 puntos porcentuales. 


\section{Journal of the Academy $|9|$}

\section{Los problemas de salud a causa de la contaminación hídrica de la Cuenca Coata}

A continuación, se presentará un análisis sobre las enfermedades más conocidas a causa de la contaminación hídrica en la cuenca Coata, las cuales son las gastrointestinales, infecciones entre otras como se puede observar (Figura 1).

Analizando las posibles enfermedades causadas por la contaminación hídrica la población afectada considera que con un 58\% (224 personas) considera que la contaminación hídrica influye en la obtención de enfermedades digestivas, el 16\% (60 personas) considera que la contaminación hídrica no afecta en la salud de la población, debido a que el consumo de agua en estas zonas es tratado o que el agua que consumen es pura ya que es del subterráneo, considerando también la ubicación de las personas encuestadas.

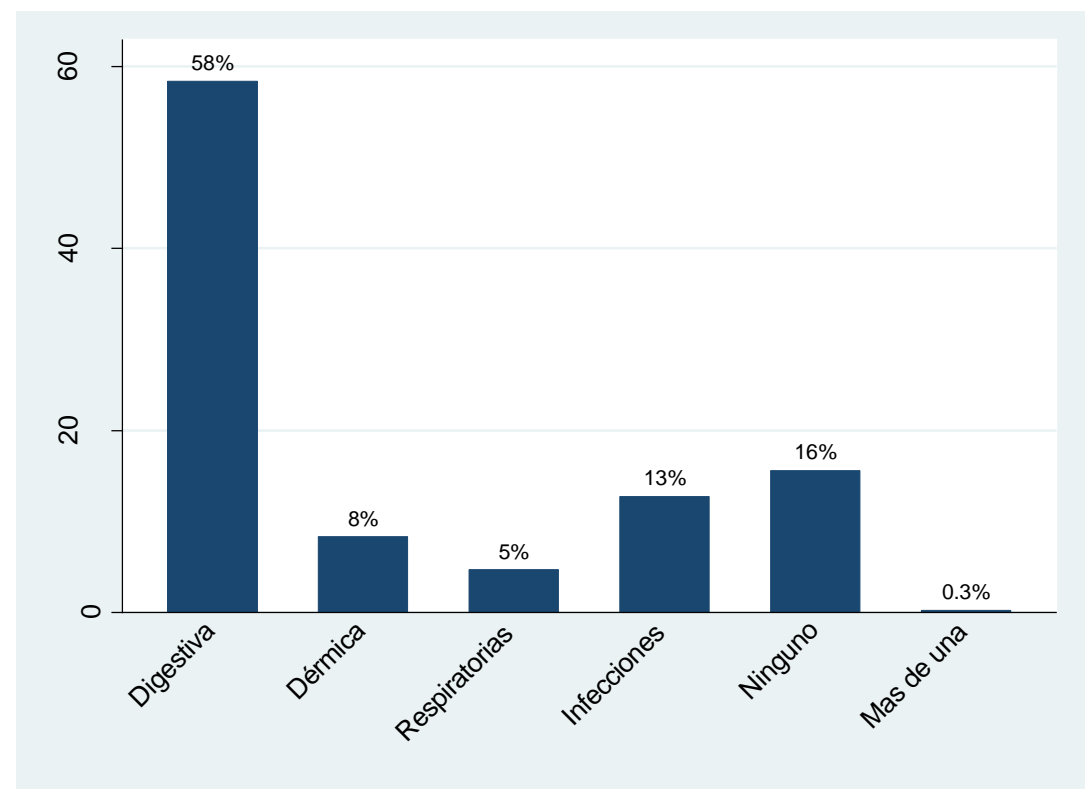

Figura 1. Enfermedades a causa de la contaminación hídrica, Elaboración propia

La repercusión de la contaminación hídrica sobre los gastos en salud (población y el gobierno).

Para el objetivo específico 3 que es "Determinar la repercusión de la contaminación hídrica sobre los gastos en salud", considerando como variable dependiente a la Salud Pública y variable independiente a la contaminación hídrica para lo cual se realizaron diferentes regresiones, para de esa forma encontrar el mejor modelo, cuyas variables independientes explique a la variable dependiente.

A continuación, se presenta la regresión y su respectiva interpretación econométrica.

Tabla 5. Resultados de la regresión logit - Objetivo específico 3

Logistic regression

$\begin{array}{llr}\text { Number of obs } & = & 384 \\ \text { Wald chi2 (1) } & = & 4.19 \\ \text { Prob > chi2 } & = & 0.0007 \\ \text { Pseudo R2 } & = & 0.2753\end{array}$

Log pseudolikelihood $=-122.05376$

Pseudo R2

0.2753

\begin{tabular}{r|rrcrrr}
\hline \multirow{2}{*}{ GS } & Coef. & $\begin{array}{c}\text { Robust } \\
\text { Std. Err. }\end{array}$ & $\mathrm{z}$ & $\mathrm{P}>|\mathrm{z}|$ & [958 Conf. Interval] \\
\hline \multirow{2}{*}{ CH } & .8206766 & .4009414 & 2.05 & 0.041 & .0348459 & 1.606507 \\
_cons & 1.547563 & .3487022 & 4.44 & 0.000 & .8641187 & 2.231006 \\
\hline
\end{tabular}




\section{Journal of the Academy $|10|$}

- El valor de "Prob>chi2 $=0.0000 "$ representa el valor de P para el test de la razón de verosimilitud que prueba la hipótesis nula $(\mathrm{H} 0)$ de que todos los coeficientes beta del modelo (los efectos) son igual a cero, tomando en consideración un nivel de confianza del $95 \%$ se acepta la hipótesis nula del modelo del objetivo específico 3.

- La interpretación del "Pseudo R2" de McFadden (similar a la interpretación del R2 tradicional) indica que aproximadamente el $27.53 \%$ de la variación de la Salud Pública (variable dependiente) puede ser explicada por la variación de la contaminación hídrica (variable independiente) del modelo. El modelo se ajusta bien cuando el pseudo R2 se aproxima a uno, en este caso específicamente el modelo no se ajusta bien debido a que el valor es menor al 0,5 se aproxima a 0 .

- Luego en la tabla se observa " $\mathrm{P}<$ lzl", se observa el valor $\mathrm{P}$ del test que prueba la hipótesis nula, de que el coeficiente de cada variable es igual a cero. En este caso, con un $95 \%$ de confianza aceptamos la $\mathrm{H} 0$ para la variable del modelo, debido a que presentan datos menores a 0.05 .

- Para la variable independiente (Contaminación hídrica), la relación con la Salud Pública es directa, debido a que el signo del coeficiente de la variable independiente $(\mathrm{CH})$ es positiva, es decir, al aumentar contaminación hídrica la población tiene mayor repercusión en su salud (mayor gasto en salud).

- El valor "z" prueba que, si la variable independiente analizada es significativa, en esta regresión específicamente se puede observar que el valor de " $z$ " es mayor a 1.96, lo que significa que la variable independiente es significativa.

De acuerdo con la siguiente tabla se muestra que la probabilidad en promedio de que la contaminación hídrica influya en la Salud Pública en la Cuenca Coata es de aproximadamente 90.43\%.

Tabla 6. Efecto marginal - Objetivo específico 3

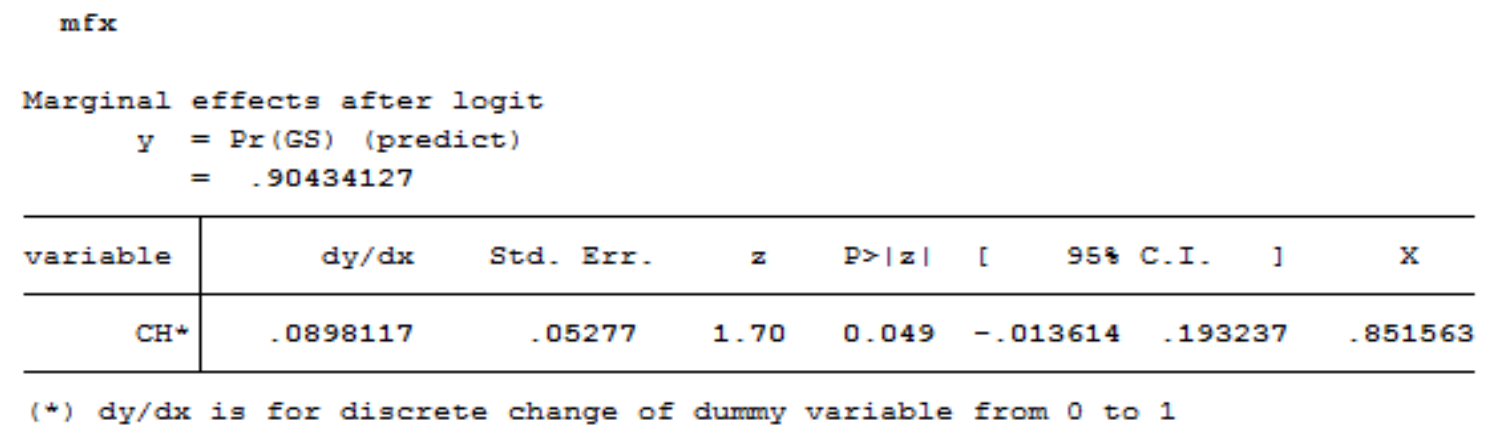

La salida del resultado nos permite ver en la columna "P>z" en donde se observa el valor $\mathrm{P}$ del test que prueba la hipótesis nula, de que el coeficiente beta de cada variable es igual a cero. En este caso, con un $95 \%$ de confianza aceptamos la $\mathrm{H}_{0}$ para la variable independiente (Contaminación hídrica), debido a que presenta un dato menor a 0.05 , luego de aplicado el efecto marginal, resulto ser significativa para el modelo.

El coeficiente resultante de la estimación del modelo refleja el efecto marginal promedio (dy/dx) de la variable independiente sobre la probabilidad de respuesta, el cual nos indica lo siguiente:

- Al existir contaminación hídrica que tengan un contacto directo o indirecto con la Cuenca Coata, la probabilidad de que influya en la salud pública incrementa en promedio 82.07 puntos porcentuales. 


\section{Journal of the Academy | $11 \mid$}

\section{Los efectos de la contaminación hídrica sobre la salud pública de la población afectada}

Para el objetivo general que es "Analizar los efectos de la contaminación hídrica sobre la salud pública de la población afectada en la cuenca Coata en el año 2019", considerando como variable dependiente la Salud Publica que será medida a través de la morbilidad y variable independiente a la contaminación hídrica, calidad de agua, información, edad, sexo y educación para lo cual se realizaron diferentes regresiones, para de esa forma encontrar el mejor modelo, cuyas variables independientes explique a la variable dependiente.

A continuación, se presenta la regresión y su respectiva interpretación econométrica.

Tabla 7. Resultados de la regresión cloglog - Objetivo general

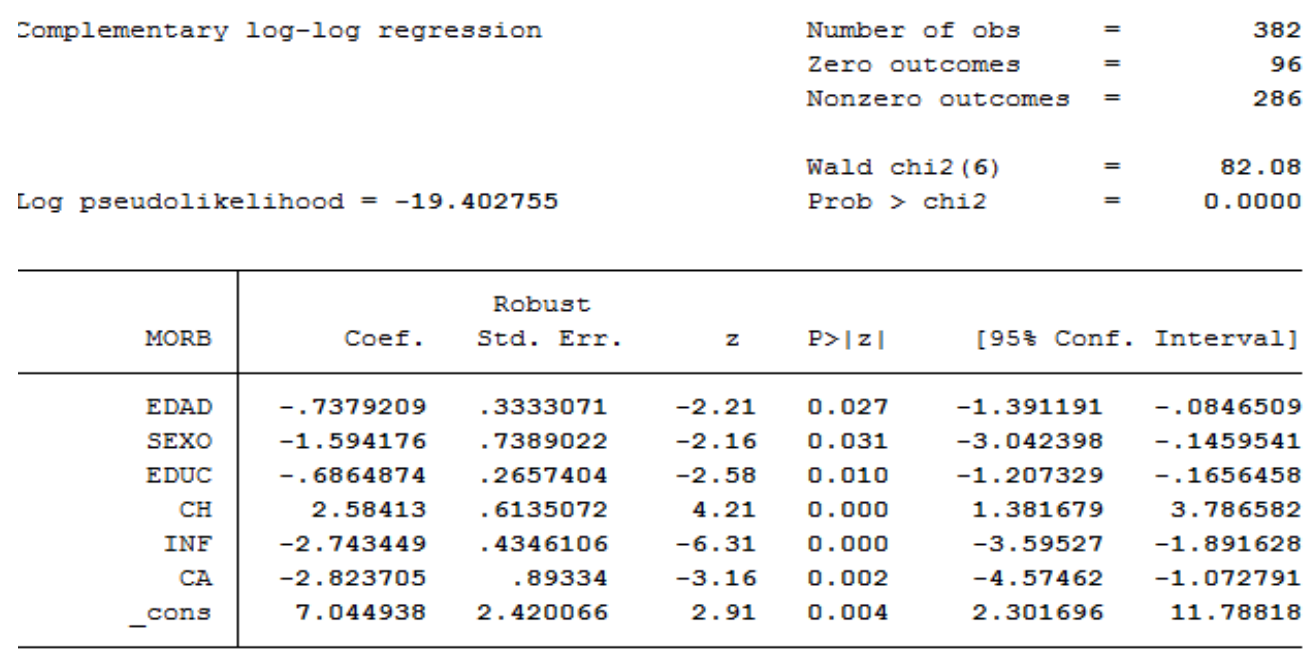

- El valor de "Prob>chi2 = 0.0000" representa el valor de P para el test de la razón de verosimilitud que prueba la hipótesis nula (H0) de que todos los coeficientes beta del modelo (los efectos) son igual a cero, tomando en consideración un nivel de confianza del 95\% se acepta la hipótesis nula del modelo general

- Luego en la tabla se observa " $\mathrm{P}<\mathrm{lzl}$ ", se observa el valor $\mathrm{P}$ del test que prueba la hipótesis nula, de que el coeficiente de cada variable es igual a cero. En este caso, con un $95 \%$ de confianza aceptamos la $\mathrm{H} 0$ para todas las variables del modelo, debido a que todas presentan datos menores a 0.05 .

- Para las variables independientes tales como la calidad del agua, información, edad, sexo y educación, la relación con la morbilidad (MORB) es inversa, debido a que los signos de los coeficientes de cada una de las variables independientes (CA, INF, EDAD, SEXO y EDUC) son negativa. Cabe resaltar que los coeficientes solo muestran la relación que tiene la variable dependiente con la variable independiente.

- Los valores " $z$ " prueban si las variables independientes analizadas son significativas, si dicho valor es mayor a 1.96 (con un nivel de confianza del 95 por ciento) prueba que cada coeficiente es diferente de cero y para rechazar dicho valor este debe de ser menor 1.96. Los valores más altos de " $z$ " significan que existe mayor relevancia de las variables, cabe resaltar que los valores del " $z$ " tienen que ser mayores a 1.96 en valor absoluto para que el modelo mantenga dichas variables (significativas). En esta regresión específicamente se puede observar que todos los valores de " $\mathrm{z}$ " son mayores a 1.96, lo que significa que todas las variables independientes son significativas.

De acuerdo con la siguiente tabla se muestra que la probabilidad en promedio de ya exista morbilidad a causa de la contaminación hídrica de la Cuenca Coata es de aproximadamente 97.91\%. 
Tabla 7. Efecto marginal - Objetivo general

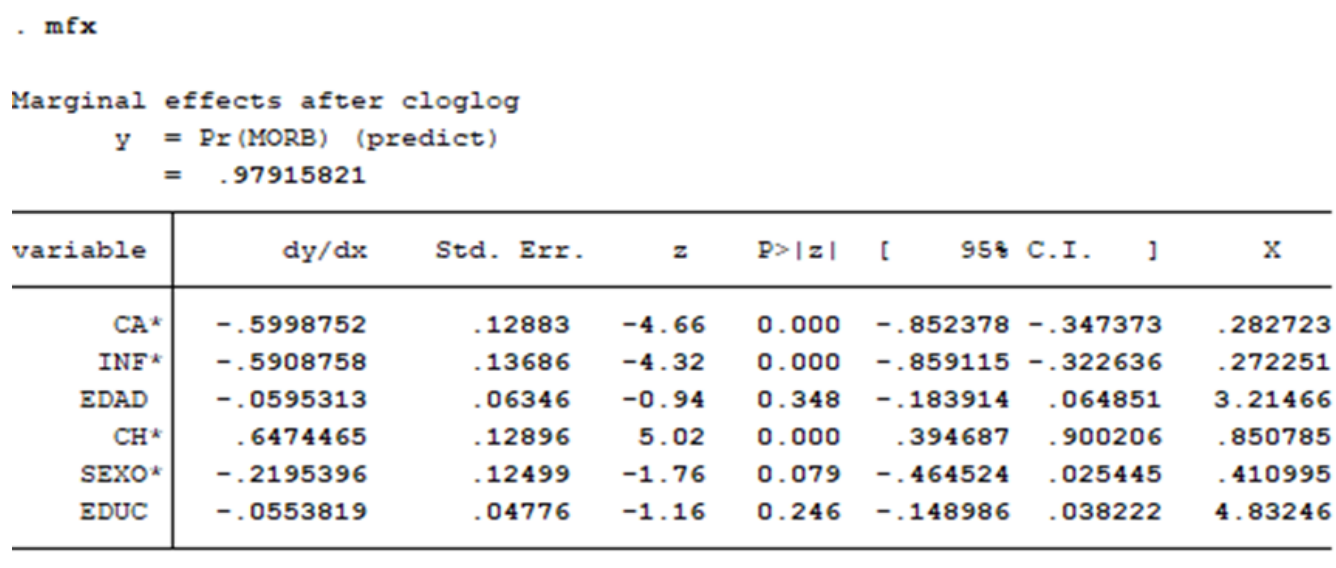

(*) $d y / d x$ is for discrete change of dummy variable from 0 to 1

La Salida de resultados nos permite ver en la columna "P>z" en donde se observa el valor $\mathrm{P}$ del test que prueba la hipótesis nula, de que el coeficiente beta de cada variable es igual a cero. En este caso, con un $95 \%$ de confianza aceptamos la $\mathrm{H}_{0}$ para las variables independientes (CA, INF y $\mathrm{CH}$ ), debido a que todas presentan datos menores a 0.05. Mientras que la edad (EDAD), sexo (SEXO) y educación (EDUC), luego de aplicado el efecto marginal, resultaron ser no significativas para el modelo.

Los coeficientes resultantes de la estimación del modelo reflejan los efectos marginales promedio $(\mathrm{dy} / \mathrm{dx})$ de cada variable independiente sobre la probabilidad de respuesta, los cuales nos indican lo siguiente:

- Al existir mayor calidad de agua (CA) la probabilidad de que exista morbilidad (MORB) a causa de la contaminación hídrica disminuirá en promedio 59.98 puntos porcentuales.

- La probabilidad de que exista morbilidad a causa de la contaminación hídrica disminuya en 59.08 puntos porcentuales cuando se cuenta con mayor información sobre el tratamiento del agua (INF).

- La probabilidad de que exista morbilidad a causa de la contaminación hídrica disminuirá en 5.95 puntos porcentuales cuando los se sea más adulto (EDAD).

- La probabilidad de que exista morbilidad a causa de la contaminación hídrica incremente en 64.74 puntos porcentuales cuando haya contaminación hídrica $(\mathrm{CH})$.

- La probabilidad de que exista morbilidad a causa de la contaminación hídrica disminuirá en 21.95 puntos porcentuales cuando sean masculinos (SEXO).

- La probabilidad de que exista morbilidad a causa de la contaminación hídrica disminuirá en 5.53 puntos porcentuales cuando se tenga más grado de instrucción educativa (EDUC).

En este sentido, analizando los resultados obtenidos en la presente investigación contrastan a investigaciones previas como la de Capacoila (2017), el cual concluye la contaminación del río Coata es provocada principalmente por la descarga incontrolada de las aguas residuales y residuos sólidos a lo largo de la ciudad de Juliaca. Efectivamente, se comprueba que esas dos variables (residuos sólidos y aguas residuales) son los principales contaminantes de la Cuenca Coata. Así mismo, el resultado se contrasta con Ocolay Laqui (2017) y Sales \&Fernández (2003).

En la investigación de Sarmiento (1999), se logró establecer la presencia de diversos contaminantes hídricos, en trece muestras de agua del embalse se encontraron metales pesados, los cuales se pueden considerar como los tóxicos más importantes a vigilar debido a que estos tienen reconocidos efectos sobre la salud humana. Por ende, en dicha investigación, el principal contaminante del recurso hídrico son los metales son los metales pesados, mientras que en nuestros resultados obtenidos se observa que, los metales pesados si es uno de los factores contaminantes del recurso hídrico, sin embargo, este no 


\section{Journal of the Academy $|13|$}

representa un porcentaje significativo. Esta diferencia encontrada entre ser y no ser un factor principal de la contaminación hídrica se debe al lugar del estudio.

En la investigación de Rodríguez, García \& García (2016) se considera que uno de los contaminantes hídricos es el uso de plaguicidas hidrofóbicos (en cantidad y frecuencia alta). Sin embargo, dentro de los resultados obtenidos de la presente investigación, no se considera significativo el uso de plaguicidas hidrofóbicos.

Según Bofill (2005), concluye que las aguas residuales son la principal fuente de microorganismos patógenos que se transmiten a través del ambiente y que llegan a la población especialmente a través de la contaminación del agua usada para beber. Contrasta los resultados que se presentan en la investigación de Bofill, sin embargo, las aguas residuales no son los únicos factores contaminantes del recurso hídrico, sino existen otros, así como los residuos sólidos, detergentes, aceites, metales pesados, etc. De acuerdo con los resultados presentados en el presente trabajo, se muestra que los residuos sólidos y las aguas residuales son los principales factores contaminantes del recurso hídrico.

Además, los estudios realizados por la Organización Mundial de la Salud (OMS) confirman que el agua contaminada genera enfermedades diarreicas agudas (EDA) y estas son la principal causa de morbilidad en familias de situación de pobreza (Fajardo, 2015).

Diferentes estudios epidemiológicos han centrado sus esfuerzos en las últimas décadas, a fin de determinar cierto tipo de relación entre la contaminación hídrica y el bienestar de las personas. Los resultados de estas diferentes investigaciones empíricas, en diversos contextos han logrado determinar un tipo de relación directa entre los niveles de contaminación hídrica y los niveles de morbilidad observados para la población. Para el caso de los países en desarrollo, la Organización Mundial de la Salud en el 2014, ha encontrado que las enfermedades asociadas con la contaminación del agua son la principal causa para la mortalidad. El Perú en su condición de país en vías de desarrollo, no ha sido la excepción a este hecho, las enfermedades diarreicas agudas (EDA) son una de las principales causas de morbilidad entre los grupos de menores ingresos principalmente (Loyola y Soncco, 2006)

En el estudio que realizo Arcilla (1998) determinó la relación la relación entre la contaminación hídrica y la morbilidad. En el cual utilizo como variable dependiente morbilidad por diarrea, y como variables independientes, la demanda bioquímica de oxígeno, ingresos, número de visitas al médico. El resultado encontrado muestra que por una disminución de $1 \%$ en la contaminación hídrica, la morbilidad de la población afectada se reducirá en un $0,14 \%$.

En observación Acosta (2017) realizó una investigación para determinar el impacto del recurso hídrico llegando de la problemática hídrica y sanitaría y su relación con enfermedades en la salud es alarmante que tiene una probabilidad $22 \%$ de que aumente el $\mathrm{N}^{\circ}$ de enfermos con un efecto marginal 0.2536. donde concluyo que la contaminación hídrica se da por mala disposición de residuos sólidos lo cual ha generado impacto en la salud pública en la región de Cauca, Colombia. Además, según el estudio de Gonzales (2014) analiza que, debido a los problemas en acceso a agua segura y a liberación de excretas, e importantes fuentes de contaminación genera un gran impacto en la salud de la población peruana, resultado que contrasta los resultados obtenidos en la presente investigación.

Así mismo según el estudio de Villena (2018) el impacto de la contaminación de fuentes hídricas llega más allá de la salud humana y abarca aspectos de producción económica y recuperación de ecosistemas, lo cual asevera en cierta proporción a los resultados obtenidos, pero indica que las consecuencias no solo se limitan a la salud pública (Davie, 2003).

Al analizar los resultados en la presente investigación, se pudo observar que los efectos de la contaminación hídrica, son explicitas mediante variables relevantes como la contaminación hídrica, ingreso, enfermedades causadas a causa del consumo de la Cuenca de Coata, acceso al agua potable. Para esto desarrollamos un modelo econométrico Probit en donde la variable dependiente es el gasto en 


\section{Journal of the Academy | $14 \mid$}

salud y las variables independientes son aquellas variables que explican en la probabilidad de que esto suceda, como se esperaba la mayor parte de variables que definimos como independientes resultaron significativas en el modelo. En una comparación de los resultados de esta investigación que al analizar la variable a una mayor contaminación hídrica el 93\% de probabilidad de aumentar el gasto en salud, a mayores ingresos hay un $26 \%$ de probabilidad de aumentar el gasto en salud, en un aumento en el acceso de agua potable hay $70 \%$ de probabilidad de que disminuya el gasto en salud. Así mismo Cardona (2003) analiza la calidad y riesgo de la contaminación del agua en la micro Cuenca del Rio La Soledad nos indica que a mayores accesos a servicios básicos hay menor probabilidad de la contaminación hídrica.

Finalmente, de acuerdo Humberto (2015), el cual concluye que la variable "Información" sobre los riesgos para la salud por causa del agua contaminada no revela ser significativa en cuanto a su relación con la morbilidad humana. Muchas familias han declarado que no existen programas de sensibilización, intensivos y continuos, acerca de los riesgos para la salud humana que conlleva la contaminación hídrica. El análisis de relaciones de causa - efecto ha mostrado relaciones importantes entre las variables de edad y género y la ocurrencia de enfermedades humanas por causa del contacto con el agua contaminada del río Vilcanota. Los signos negativos indicarían que cuando los jefes de familias son personas de edad mayor y sexo masculino, es menor la probabilidad de presencia de estas enfermedades.

De acuerdo a la teoría del beneficio marginal de reducción en la contaminación está dado por los ahorros en los gastos defensivos necesarios para mantener la Calidad del Ambiente Personal constante. Este modelo fue demostrado por Araujo (2003) donde el beneficio marginal de reducción de la contaminación es igual al ahorro en los gastos defensivos. Donde la salud familiar deseada que depende de las condiciones ambientales de la localidad y que afecta directamente la utilidad del hogar (Balmes, 2006).

\section{CONCLUSIONES}

Los determinantes que incrementan la probabilidad de la existencia de la contaminación hídrica se dan cuando los residuos sólidos y aguas residuales entran en contacto con las aguas de la Cuenca Coata. Por un lado, la probabilidad de que exista contaminación hídrica incrementa en 19.15 puntos porcentuales cuando las aguas residuales tienen contacto con el rio de la Cuenca Coata. Mientras que, al existir residuos sólidos que tengan un contacto directo o indirecto con la Cuenca Coata, la probabilidad de que exista contaminación hídrica incrementa en promedio 99.96 puntos porcentuales, esta última es la que explica en mayor porcentaje a la variable dependiente (contaminación hídrica).

Los principales problemas de salud pública a causa de la contaminación hídrica de la cuenca Coata son gastrointestinales ya que un 58\% de los encuestados señalo que era la principal consecuencia del consumo de agua de baja calidad son las digestivas y el $16 \%$ de los encuestados considera que la contaminación hídrica no afecta en la salud de la población, debido a que el consumo de agua en estas zonas es tratada o pura, esto se debe a la falta de información sobre efecto de la contaminación hídrica los cuales se verá a largo plazo.

La contaminación hídrica a causa de factores y agentes contaminantes genera un impacto directo y negativo a la salud pública lo cual permite reflejar la situación actual de la Cuenca del Rio Coata y de la población en contacto que deben merecer la atención de las autoridades de salud del país, considerando la participación integra de las instituciones involucradas y la participación de la población afectada, para llegar al desarrollo sostenible de los lugares afectados.

Finalmente, las variables con mayor influencia sobre la probabilidad de morbilidad humana generada por enfermedades de origen hídrico, son la calidad de agua, información y la contaminación hídrica, es decir si una familia cuanta, con una buena calidad de agua e información para su tratamiento, es baja la probabilidad de que se enferme mientras que si incrementa la contaminación hídrica la probabilidad de morbilidad es mayor. Las variables no tan significativas pero importantes son la edad del jefe de familia 


\section{Journal of the Academy | $15 \mid$}

educación y el género. Esto significa que se puede esperar una menor presencia de enfermedades de este tipo en los hogares rurales en los cuales el jefe de familia es de edad madura con grado de instrucción y es de género masculino. Estos resultados se obtuvieron mediante el análisis econométrico, utilizando un modelo CLOGLOG de elección discreta, los resultados mencionados tienen coeficientes de significación al $5 \%$.

\section{REFERENCIAS BIBLIOGRÁFICAS}

Acosta, M. (2017). Identificación de impactos ambientales generados por actividades económicas dentro de la reserva forestal protectora la sierra en la vereda pontezuela del municipio de santa rosa de osos Antioquia en el marco del programa manos a la paz del PNUD y alianza. Bogotá D.C.: Universidad Distrital Francisco José de Caldas.

Amaya, L. (2005). La gestión intermunicipal del agua en México. doi:10.4000/vertigo.9692

Araujo, A. (2003). Evaluación de las comunidades fitoplanctónicas en la laguna de Moronachocha (Loreto - Perú), durante el periodo creciente. Obtenido de http://repositorio.unapiquitos.edu.pe/handle/UNAP/4946

Arcilla. (1998). Determinantes de los efectos en la salud por contaminantes hídricos. Tesis de magíster en Economía del Medio Ambiente y de los Recursos Naturales. Universidad de los Andes, Bogota.

Bofill S., C. P. (2005). Efectos sobre la salud de la contaminación de agua y alimentos por virus emergentes humanos. Madrid: Unversidad de Barcelona.

Capacoila, J. (2017). EVALUACIÓN DE LA CONCENTRACIÓN DE METALES PESADOS EN LAS AGUAS SUPERFICIALES DEL RIO COATA.

Cardona, A. (2003). Calidad y riesgo de contaminación de las aguas superficiales en la microcuenca del Rio La Soledad. Obtenido de http://orton.catie.ac.cr/repdoc/A0118e/A0118e.pdf Cazau, P. (2006). Fundamentos de Estadística. Buenos aires: UBA.

CENRE. (1999). Departamento de Economía y Centro de Economía de los recursos naturales y el medio ambiente. Obtenido de Beneficios económicos de una reducción de la contaminación atmosférica en Santiago de Chile: http://www.scielo.org.mx/pdf/ineco/v59n227/0185-1667-ineco-59-227-143.pdf

Comisión Económica del Agua. (2011). Sistema de cuencas ambientales y económicas del agua. Obtenido de https://repositorio.cepal.org/bitstream/handle/11362/3148/1/SCAE-Agua03-082011_FINAL_es.pdf

Davie, T. (2003). Hidrology as a Science. Fundamentals of hidrology. Londres: Routledge.

Encarnación, F. (1985). Introducción a la flora y vegetación de la Amazonía Peruana: Estado actual de los Estudios, medio natural y ensayo de una calve de determinación de las formaciones vegetales en la llanura Amazónica. Obtenido de http://www.iiap.org.pe/upload/publicacion/DT012.pdf

Fajardo, D. (2015). Análisis de impactos ambientales y factores impactantes en la microcuenca Buenos Aires zona rural del municipio de Lorica (Córdoba). Manizales Caldas: Universidad de Manizales.

Gonzales, G., \& Zevallos, A. (2014). Contaminación ambiental, variabilidad climática y cambio climático. una revisión del impacto en la salud de la población peruana. Obtenido de http://www.scielo.org.pe/pdf/rins/v31n3/a21v31n3.pdf

Hernández Sampieri, R., Fernández Collado, C., \& Baptista Lucio, P. (2010). Metodología de la investigación. 


\section{Journal of the Academy | $16 \mid$}

Humberto, C. (2015). Contaminación Del Agua Y Pobreza Rural: El Caso De La Cuenca Alta Del Río Vilcanota. Cusco. Universidad Nacional Agraria La Molina, 1-100.

Kerlinger, F. (1979). Enfoque conceptual de la investigación del comportamiento: Técnicas y Metodologías. México: Nueva Editorial Interamericana.

Loayza, J., \& Cano, P. (2015). Impacto de las actividades antrópicas sobre la calidad del agua de la subcuenca del rio Shullcas. Obtenido de http://repositorio.uncp.edu.pe/bitstream/handle/UNCP/3507/Loayza\%20Quispe\%20-

$\% 20$ Cano\%20Rojas.pdf?sequence $=1 \&$ isAllowed $=\mathrm{y}$

Loyola, R. y Soncco, C. ( 2006). Valoración económica del efecto en la salud por cambio en la calidad del agua en zonas marginales de Lima y Callao. CIES. Universidad Nacional Agraria de La Molina. Lima. PE.

Mayta, S. (1999). Contaminación del agua. Obtenido de https://docs.google.com/viewer?a=v\&pid=sites\&srcid=ZGVmYXVsdGRvbWFpbnxjb250YW1pbmFj aW9uZGVsYXxneDpjOWM3YzVhYWFiNmRIYWI

Ocola. J y Laqui. W. (2017). Fuentes contaminantes de la cuenca del titicaca,Un aporte al conocimiento de las causas que amenazan la calidad del agua del maravilloso lago Titicaca. Autoridad Nacional del Agua, ISBN: 978-612-4273-12-4. Resuperado de: http://www.minam.gob.pe/puno/wpcontent/uploads/sites/55/2014/02/ESTUDIO-DEL-ESTADO-DE-LA-CALIDAD-AMBIENTALCUENCA-DEL-TITICACA..pdf.

Olmeda, M. (2006). El agua y su análisis desde la perspectiva económica: una aplicación para el crecimiento económico. Obtenido de http://altea.daea.ua.es/ochorem/comunicaciones/MESA2COM/OlmedaPascualJoseMiguel.pdf Ravelo, M. C., \& Balaguer, E. P. (2019). Funciones de enlace alternativas en modelos de respuesta binomial. Economía, 44(48), 9-35.

Rodriguez Miranda, J., García Ubaque, C., \& García Ubaque, J. (2016). enfermedades transmitidas por el agua y saneamiento básico en colombia. Bogotá, Colombia. doi:http://dx.doi.org/10.15446/rsap.v18n5.54869

Sales, M., \& Fernandez, J. (2003). Diccionario Mosby de medicina, enfermería y ciencias de la salud. Madrid: Elsevier España, S.A.

Samboni N, Carvajal Y \& Escobar J. (2007). Revisión de parámetros fisicoquímicos como indicadores de la calidad y contaminación del agua. Rev. Ingeniería e investigación vol 27 núm 3. 172-181 p.

Sarmiento M., I. Á. (1999). Evaluación del impacto de la contaminación del embalse de Muña sobre la salud humana. Revista de salud pública.

Villena, J. (2018). Calidad del agua y desarrollo sostenible. Lima: Universidad Nacional de Ingeniería.

Zegarra, E. (2014). Economía de agua. Lima: Grupo de Análisis para el Desarrollo (GRADE). 